\title{
NARROW DUST JETS IN A DIFFUSE GAS COMA: A NATURAL PRODUCT OF SMALL ACTIVE REGIONS ON COMETS
}

\author{
M. R. Combi, V. M. Tenishev, M. Rubin, N. Fougere, and T. I. Gombosi \\ Department of Atmospheric, Oceanic and Space Sciences, University of Michigan, 2455 Hayward Street, Ann Arbor, \\ MI 48109-2143, USA; mcombi@umich.edu \\ Received 2011 November 1; accepted 2012 February 1; published 2012 March 19
}

\begin{abstract}
Comets often display narrow dust jets but more diffuse gas comae when their eccentric orbits bring them into the inner solar system and sunlight sublimates the ice on the nucleus. Comets are also understood to have one or more active areas covering only a fraction of the total surface active with sublimating volatile ices. Calculations of the gas and dust distribution from a small active area on a comet's nucleus show that as the gas moves out radially into the vacuum of space it expands tangentially, filling much of the hemisphere centered on the active region. The dust dragged by the gas remains more concentrated over the active area. This explains some puzzling appearances of comets having collimated dust jets but more diffuse gaseous atmospheres. Our test case is $67 \mathrm{P} /$ Churyumov-Gerasimenko, the Rosetta mission target comet, whose activity is dominated by a single area covering only $4 \%$ of its surface.
\end{abstract}

Key words: acceleration of particles - comets: general - comets: individual (67P/Churyumov-Gerasimenko)

\section{INTRODUCTION}

When comets are active in the inner solar system, they often exhibit a broadly distributed gas coma (a comet's tenuous atmosphere) but more narrowly collimated dust jets. This is seen in images taken during spacecraft flybys such as 1P/Halley by Giotto (Keller 1990), in 9P/Tempel 1 (Figure 1) by Deep Impact (A'Hearn \& Combi 2007), in 103P/Hartley 2 by the EPOXI mission (A'Hearn et al. 2011), in the narrow beams of dust seen in the Deep Space 1 images of comet 19P/Borrelly (Soderblom et al. 2004), as well as many Earth-based remote observations.

The coma of gas and dust from most comets is driven by sublimation of frozen ice at or near the surface from only a fraction of the surface exposed to the sun. While the recent EPOXI mission target comet 103P/Hartley 2 (A'Hearn et al. 2011; Combi et al. 2011), 46P/Wirtanen (Rickman \& Jorda 1998), 73P/Schwassmann-Wachmann 3 (Toth et al. 2005), and 1996 B2 Hyakutake (Lisse et al. 1999; Combi et al. 2005) seem to require large total active surface areas, the active surface fractions of most comets are in the 5\%-20\% range. In fact, the surprising EPOXI results could indicate that comets described as having a large active fraction may produce most of their gas from released icy grains. There is certainly evidence for this in 73P and Hyakutake.

Comet 67P/Churyumov-Gerasimenko is a Jupiter Family Comet and the target comet of the Rosetta mission (Schulz 2009), which was launched on 2004 March 2 and will make a rendezvous and landing in the latter part of 2014. There will be an extensive monitoring of the nucleus and coma during the following year through its most active period around perihelion. The comet was discovered in 1969 (Churyumov et al. 1969). It has a period of 6.5 years, and its perihelion distance during the 2009 apparition was 1.25 AU. Although 67P has been observed during a few apparitions since its discovery, it has become a subject of much more interest since 2002 when it was chosen to be the back-up target for the Rosetta mission after the initial launch to comet 46P/Wirtanen was cancelled as a safety precaution. The nucleus size was determined from Hubble Space Telescope observations to have a mean radius of $\sim 2 \mathrm{~km}$ (Lamy et al. 2007). It has been characterized compositionally as a slightly depleted comet in the A'Hearn et al. (1995) and Fink (2009) ground-based surveys of the production of the common "visible" radicals $\left(\mathrm{C}_{2}, \mathrm{C}_{3}, \mathrm{CN}, \mathrm{NH}\right.$, and $\left.\mathrm{NH}_{2}\right)$ compared with water. Fink (2009) has characterized it in a subgroup called the 9P/Tempel 1 type.

Agarwal et al. (2007) summarized a number of observations of the comet, principally from the 1982/1983, 1996/1997, and 2002/2003 apparitions with the intent of characterizing expectations of gas and dust production in advance of the Rosetta spacecraft encounter and rendezvous. The maximum water production rates, normally in the two to three weeks after perihelion, have been between 0.7 and $2.0 \times 10^{28} \mathrm{~s}^{-1}$ in the apparitions observed since 1982 (Hanner et al. 1985; Feldman et al. 2004; Mäkinen 2004; Crovisier et al. 2002) and the maximum values of the dust parameter, $\operatorname{Af} \rho$, have been in the range of 500-1400 (Storrs et al. 1992; Osip et al. 1992; A'Hearn et al. 1995; Kidger 2003; Lamy et al. 2003; Weiler et al. 2004; Schulz et al. 2004; Feldman et al. 2004; Schleicher 2006), which is fairly dusty.

Schleicher (2006) presented and analyzed a set of photometric observations of gas and dust in 67P from the 1982/1983 and $1995 / 1996$ apparitions covering a time period from two months before to four months after perihelion. It was found that the main source region of activity corresponds to only about $3 \%-4 \%$ of the total surface area of the nucleus. That this source region is generally located in one region on the surface is consistent with both the substantial rotationally driven variable activity at some times during its orbit and also by an overall strong seasonal effect, with the pre-perihelion activity being much lower than the post-perihelion activity. Groussin et al. (2007) constructed a model that accounted for the pole orientation, rotation period, and the size and location of active regions to reproduce water production rate variation. Similarly, they found that one very small active region that covers only $4 \%-5 \%$ of the surface could match their observations and that the spot is located in the north at a latitude of about $65^{\circ}$. They also found that two small active regions were not impossible, but less likely. A larger, low activity region could fit the data, but only the portion of this region in the northern hemisphere could explain the production rate at perihelion. The seasonal effect is such 


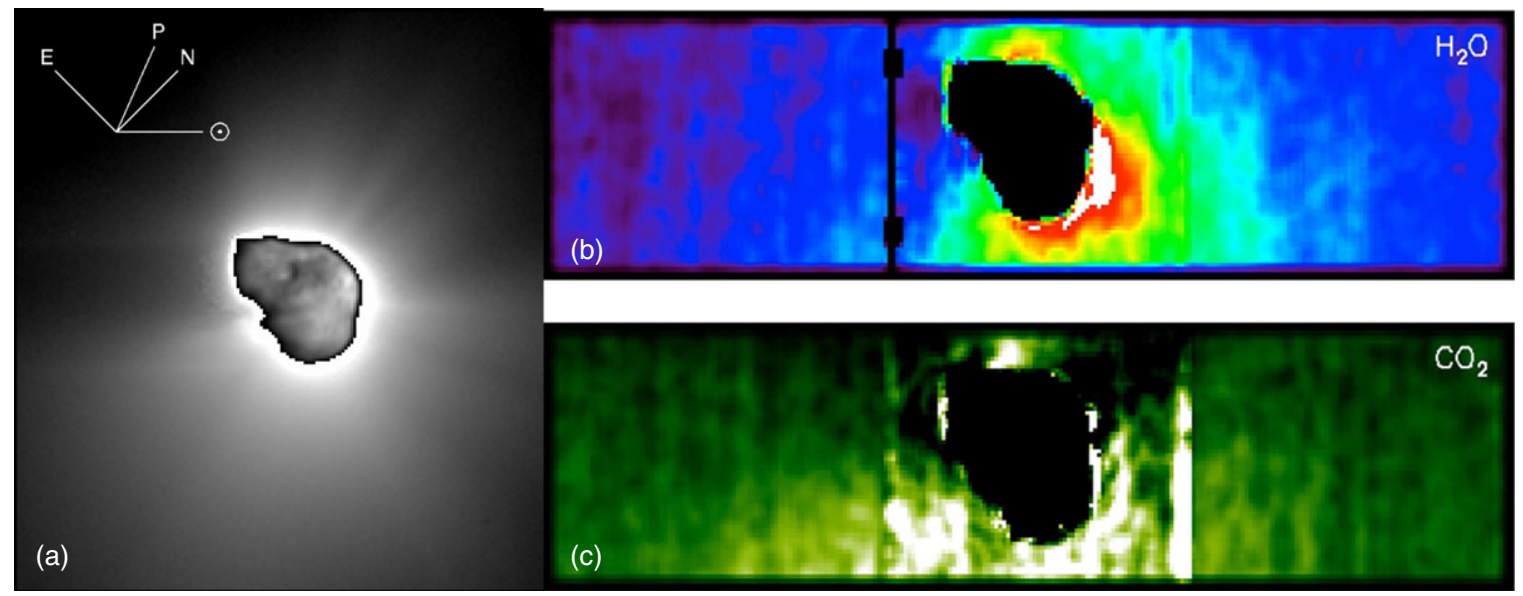

Figure 1. Diffuse gas distribution and narrow dust jet distribution in comet 9P/Tempel 1 taken by the Deep Impact spacecraft in 2005 (Farnham et al. 2007 ; Feaga et al. 2007). Panel (a) shows a white light image showing the narrow dust jets, panel (b) shows the diffuse $\mathrm{H}_{2} \mathrm{O}$ gas distribution, and panel (c) shows the different but still diffuse $\mathrm{CO}_{2}$ gas distribution. Images were taken from the Deep Impact mission Web site, http://deepimpact.umd.edu/gallery/images-results.html.

that the active region comes predominantly into the summer hemisphere around perihelion after being predominantly in the winter hemisphere before perihelion. The combination of rotationally driven and a strong seasonal effect in both gas and dust points to the major source of gas and dust in the comet to originate from this one small active region. In addition, the overall level of the water production rate is also consistent with $4 \%$ of the total surface area being active.

While there have been significant advances in both hydrodynamic and kinetic approaches to simulate the physics of the cometary coma beyond one-dimensional spherical models, these have been applied only to broadly distributed activity with dominant day/night asymmetries (Körösmezey \& Gombosi 1990; Combi 1996; Combi et al. 1997, 2004; Crifo \& Rodionov 1997, 2000). The flow and the structure of a coma produced by a small active region on a nucleus have not been explored.

This paper addresses such a case using a kinetic Direct Simulation Monte Carlo (DSMC) approach (Tenishev et al. 2008, 2011) because 67P is a fairly weak comet and explores the conditions ranging from when the comet is far from the Sun, corresponding to the rendezvous and lander phases of the Rosetta mission, to when the comet is active near perihelion. In the remainder of this paper (1) the DSMC method is briefly described, (2) the model results for the gas distributions of four cases of a small active area are compared with a previous study of a broadly distributed source (Tenishev et al. 2008), (3) the effects of a small versus broad distribution on the appearance of the $\mathrm{OH}$ coma are shown, which should be similar to typical radicals, $\mathrm{CN}$ and $\mathrm{C}_{2}$, (4) the model results for the dust distribution near the nucleus for a small active area are described (Tenishev et al. 2011), and (5) a summary of the results is presented and implications are discussed for some general observed properties of comets.

\section{DSMC MODEL}

Kinetic rather than hydrodynamic approaches to modeling cometary comae recognize that not enough collisions occur between molecules in the coma to maintain thermal equilibrium in important regions (Combi et al. 2004). Kinetic models based on the DSMC method (Bird 1994) have been applied to simulate the coma (Combi 1996; Tenishev et al. 2008, 2011; Bird 1994; Harris et al. 1997; Skorov et al. 2004; Crifo et al. 2005; Zakharov et al. 2009; Davidsson et al. 2010)
Table 1

DSMC Model Parameters/Assumptions

\begin{tabular}{|c|c|c|}
\hline Composition & \multicolumn{2}{|c|}{$95 \% \mathrm{H}_{2} \mathrm{O}, 5 \% \mathrm{CO}$} \\
\hline Photodecomposition & \multicolumn{2}{|c|}{$\mathrm{H}_{2} \mathrm{O}$ to $\mathrm{OH}, \mathrm{H}_{2}, \mathrm{H}$, and $\mathrm{O}$} \\
\hline Nucleus build density & \multicolumn{2}{|c|}{$300 \mathrm{~kg} \mathrm{~m}^{-3}$} \\
\hline Dust/gas production ratio & \multicolumn{2}{|c|}{0.8} \\
\hline Dust particle size distribution & \multicolumn{2}{|c|}{$Z(a) \sim a^{-4}$} \\
\hline Production distribution & \multicolumn{2}{|c|}{$95 \%$ from a $4 \%$ spot } \\
\hline Global regime & \multicolumn{2}{|c|}{$r=2 \mathrm{~km}$ to $r=10^{6} \mathrm{~km}$} \\
\hline \multicolumn{3}{|l|}{ Four cases } \\
\hline & $r(\mathrm{AU})$ & $Q\left[\mathrm{H}_{2} \mathrm{O}\right]\left(\mathrm{s}^{-1}\right)$ \\
\hline & 1.29 & $5 \times 10^{27}$ \\
\hline & 2.0 & $8 \times 10^{26}$ \\
\hline & 2.7 & $8 \times 10^{25}$ \\
\hline & 3.25 & $1 \times 10^{24}$ \\
\hline
\end{tabular}

in the transition and collisionless regimes, where the mean free path of molecules is too large for hydrodynamics to be applicable. The gas is modeled as a set of molecules as they move around within a grid, colliding with other molecules and dust particles. Properties, such as density, velocity, and temperature, are computed by performing standard kinetic theory averaging of particle masses, locations, velocities, and internal energies over the particle distribution function. The DSMC method is based on the rarefied-gas assumption, where over a short time interval, molecular motion and intermolecular collisions are uncoupled and can be calculated independently. Molecules are moved over the distances for this time step, and then a set of representative collisions are computed. The DSMC comet model (Tenishev et al. 2008, 2011) used here is general and has also been applied to the plumes of Enceladus (Waite et al. 2006; Tenishev et al. 2010) and Mars' exosphere (Valeille et al. 2009).

DSMC calculations for a dusty-gas coma were performed for four cases with a dominant single active region covering $4 \%$ of the surface of an assumed spherical nucleus of 67P with a radius of $2 \mathrm{~km}$. For comparison with a broad dayside dominated coma the conditions and assumptions were adopted from previous publications (Tenishev et al. 2008, 2011), which describe in detail the formulations and procedures for the gas kinetics and dust-gas interactions, respectively. The model parameters are summarized in Table 1. For the small active area $95 \%$ of the gas and dust production was emitted from a single spot 
covering $4 \%$ of the surface area with the remaining $5 \%$ distributed on the remaining $96 \%$ of the surface in proportion to that in previous calculations (Tenishev et al. 2008). The $4 \%$ active area was centered on the symmetry axis and the calculations were performed assuming axial symmetry. The coma environment for such an assumed single active region is more efficiently calculated assuming two-dimensional cylindrical axial symmetry without loss of generality.

\section{GAS COMA}

DSMC calculations for a global dusty-gas coma were performed for four cases with a dominant single active region covering $4 \%$ of the surface of an assumed spherical nucleus for $67 \mathrm{P}$ with a radius of $2 \mathrm{~km}$ (Figure 2). The determinative effects of a dominant small active area occur within the first $50 \mathrm{~km}$ of the nucleus. Figure 3 shows false color plots of the water density distribution within 100-200 km from the center of the nucleus obtained for the four cases $(1.29,2.0,2.7$, and 3.25 AU) comparing the $4 \%$ small active area with a mostly dayside-distributed source done previously (Tenishev et al. 2008). What is remarkable is that although nearly all the gas emission originates from the surface within a cap extending only $23^{\circ}$ from the center of the active area, the gas coma fills nearly the entire hemisphere centered on the active area by 10-20 times the nucleus radius in all four cases.

In the perihelion case, the gas conditions near the nucleus are in a hydrodynamic regime, which the DSMC method computes correctly despite its general applicability. Here the lateral pressure causes the gas to expand both radially and tangentially, so the flow is non-radial near the surface from the edges of the active area, as seen in the streamlines in Figure 4. As the coma becomes thinner for the other cases, the lateral expansion happens as the conditions become more and more like free molecular flow, which is essentially reached in the 3.25 AU case.

The expansion of the flow is illustrated quantitatively in Figure 5. The water density is plotted as a function of angle from the center of the active area and compares the small active area and broad sources at distances ranging from just above the surface of the nucleus $(2 \mathrm{~km})$, and at $2.5,5$, and $50 \mathrm{~km}$ from the center of the nucleus. The night side emission for the small active area models is much less than that from the broad-source models (Tenishev et al. 2008) by way of the assumed conditions at the nucleus.

\section{APPEARANCE OF DAUGHTER SPECIES}

Previous work (Tenishev et al. 2008) showed the modeled appearance of the $\mathrm{H}_{2} \mathrm{O}$ and $\mathrm{OH}$ coma for the "broadly distributed" source, as would be viewed for a typical ground-based observer for the geometry of the 2009 apparition of comet $67 \mathrm{P}$ assuming a Sun-comet-observer angle of $35^{\circ}$. Figure 6 shows the modeled $\mathrm{OH}$ coma for the four cases for both the broadly distributed and $4 \%$ small active region sources for a $35^{\circ}$ angle between the line of sight and the orientation of the center of the active area. The respective halves of the coma containing the activity, whether broadly distributed or concentrated in the small $4 \%$ source, are nearly the same. This is not surprising since the water distribution on the hemisphere with the active area is only different within about $20 \mathrm{~km}$ from the nucleus.

This comparison is not meant to be literally predictive of an actual observation, because a $4 \%$ active area on $67 \mathrm{P}$ would be at some latitude and longitude with respect to the comet's spin axis

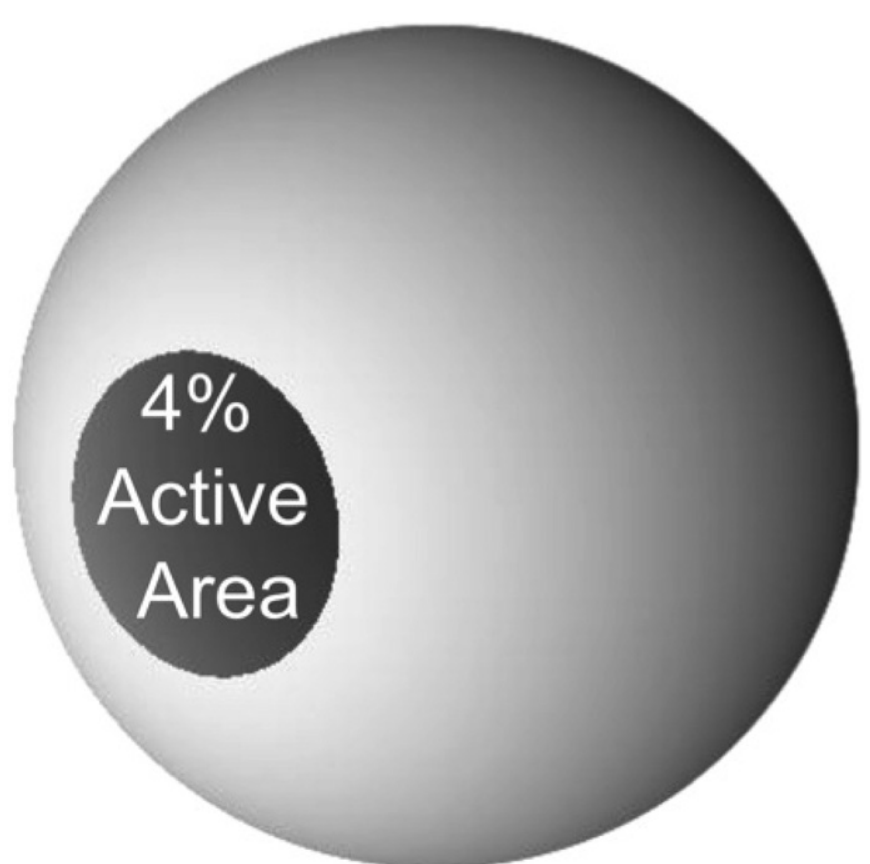

Figure 2. 4\% active area spot on 67P/Churyumov-Gerasimenko as a sphere. This is a simple illustrative picture to show the small size of a $4 \%$ active area compared with an idealize sphere.

on the surface of the rotating nucleus and varying its activity with illumination by solar light in time. The rotation period is significant compared with the transport time of both parent molecules and daughter radicals to the typical observable coma. These real effects of rotation of the nucleus will contribute to making the resulting $\mathrm{OH}$ comae appear even more circular than these static models because of the variation of the position of the active region with respect to the line of sight.

More importantly the real appearance of a comet with a small isolated active area will be complicated by a number of other effects. The combination of the initial tangential expansion, filling almost an entire hemisphere in the inner coma, with rotational and line-of-sight projection effects and "vectorial" ejection of daughter species through exothermic photodissociation makes an observed $\mathrm{OH}$ coma distribution (or $\mathrm{CN}, \mathrm{C}_{2}$, etc.) appear much more circular than might otherwise be expected. To appear like Figure 6 would require an active region close to the spin axis that would also be nearly perpendicular to the line of sight. The major finding is that the resulting gas coma for a small active spot and broadly hemispherical active region will yield comae observed from a distance that will not be very distinguishable from one another.

\section{DUST TRANSPORT}

The dust distribution was computed only out to $\sim 200 \mathrm{~km}$ from the nucleus. To calculate the dust distribution to much larger distances would require time-dependent 3D calculations of orbital trajectories of dust in a comet-Sun system with explicit time-dependent variable gas and dust production from the nucleus. By $\sim 20 \mathrm{~km}$ from the center of the nucleus, all dust particles reach their terminal velocities via gas drag. Large dust particles are accelerated to fairly slow velocities (several meters per second) and thus transport times in the coma can be very long: from days to weeks to months. The dust parameters used are described in Table 1 . Because the production rates are rather low for $67 \mathrm{P}$, even at perihelion, there is little physical 

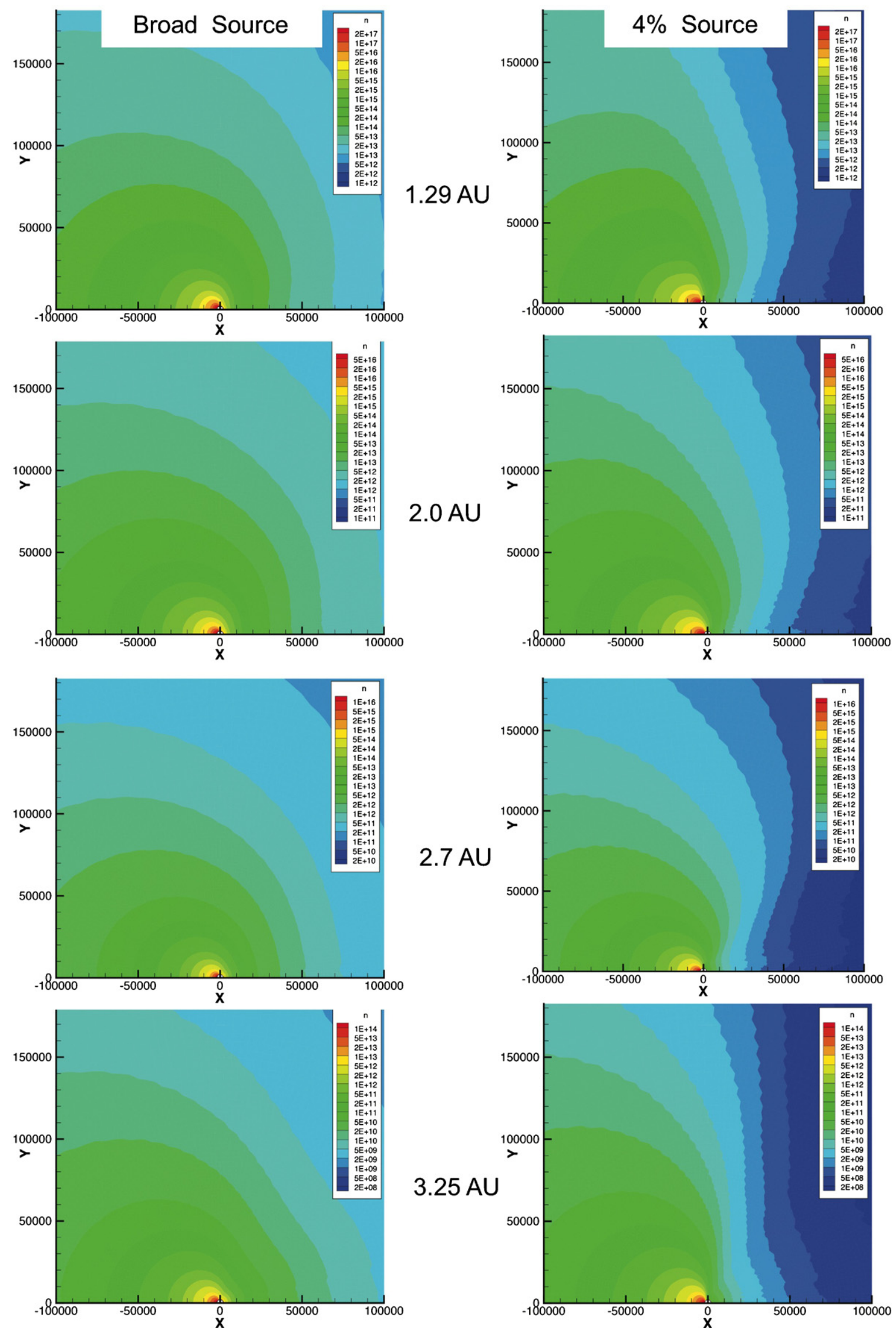

Figure 3. Distribution of water vapor in the inner $100 \mathrm{~km}$ of the coma. The water density distribution comparing the broad hemispherical source (left) with the small $4 \%$ active area (right). The color legend gives the density in $\mathrm{m}^{-3}$. Distances are in meters. Panels from the top down are for heliocentric distances of $1.29,2.0,2.7$, and $3.25 \mathrm{AU}$, respectively. 


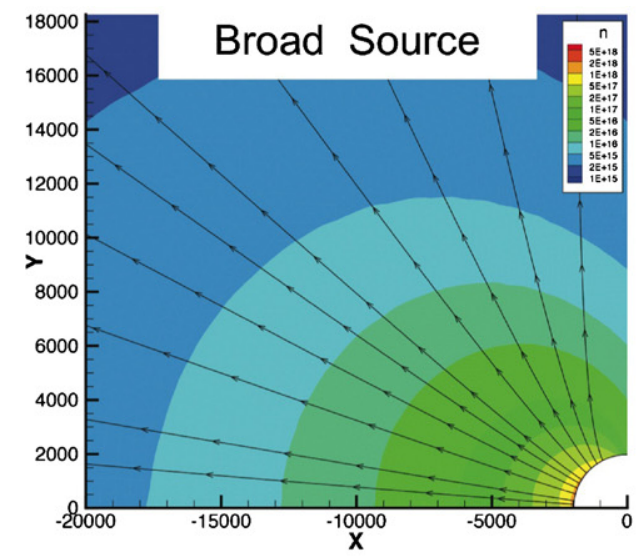

$1.29 \mathrm{AU}$
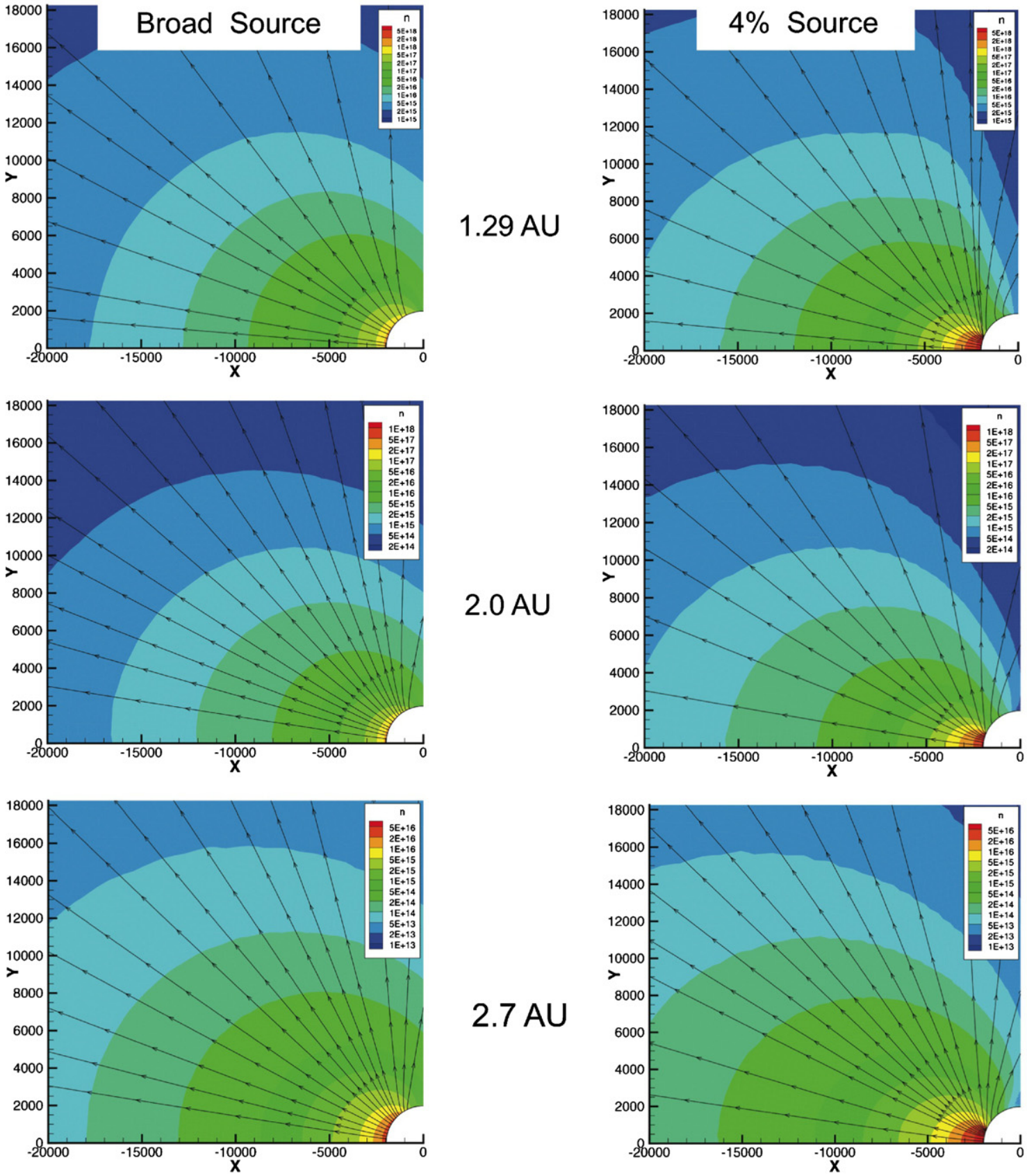

$2.0 \mathrm{AU}$

\section{$2.7 \mathrm{AU}$}
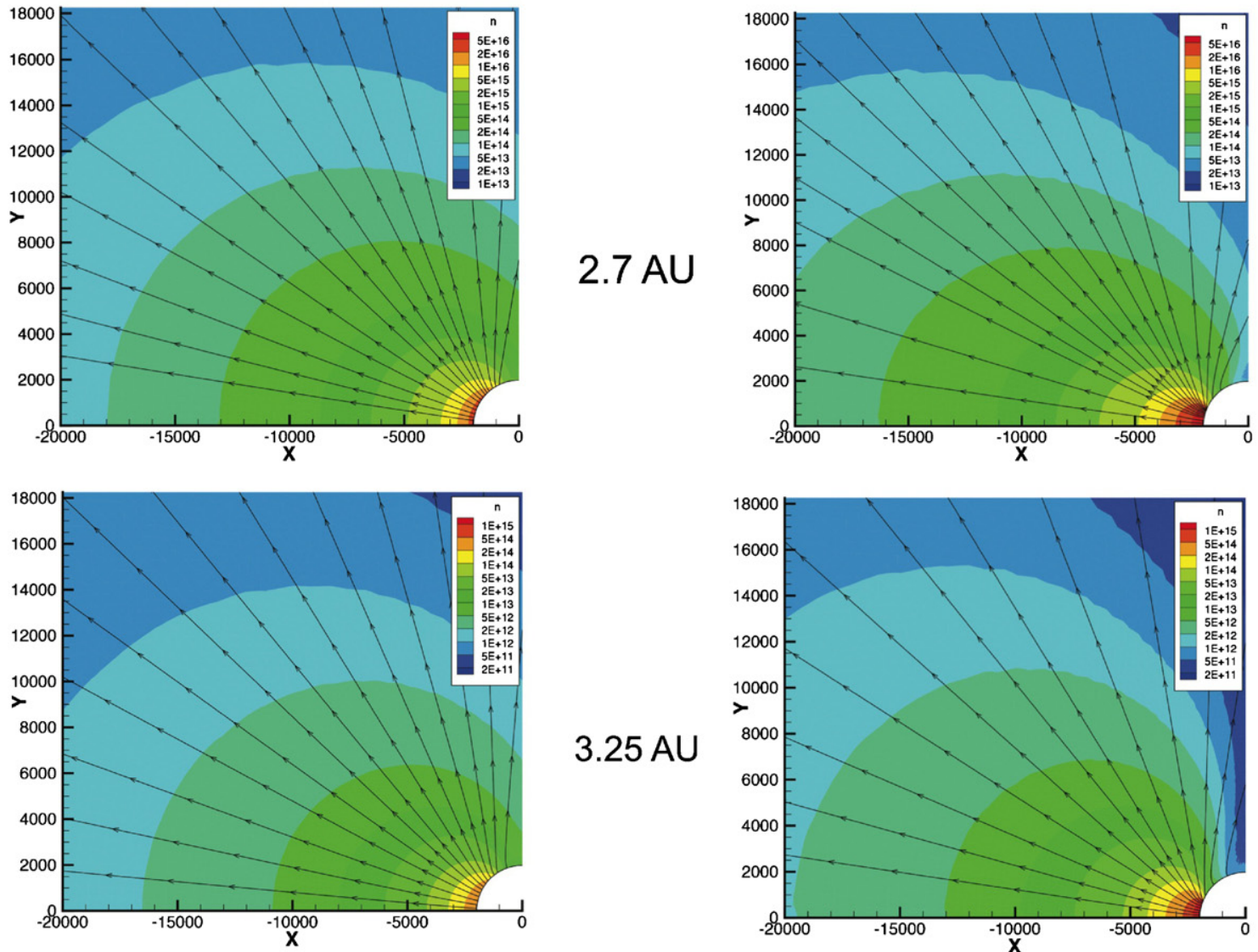

Figure 4. Water vapor density distribution in the very inner coma. Shown (left) are water vapor density contours in false color and flow streamlines for the inner $20 \mathrm{~km}$ region of the coma for the broadly distributed source (Tenishev et al. 2008) compared with that from a $4 \%$ area source (right). The plots from top to bottom are for heliocentric distances of $1.29,2.0,2.7$, and $3.25 \mathrm{AU}$, respectively. The quarter white circles in the lower right of each panel correspond to a spherical $2 \mathrm{~km}$ radius nucleus. 
$1.29 \mathrm{AU}$

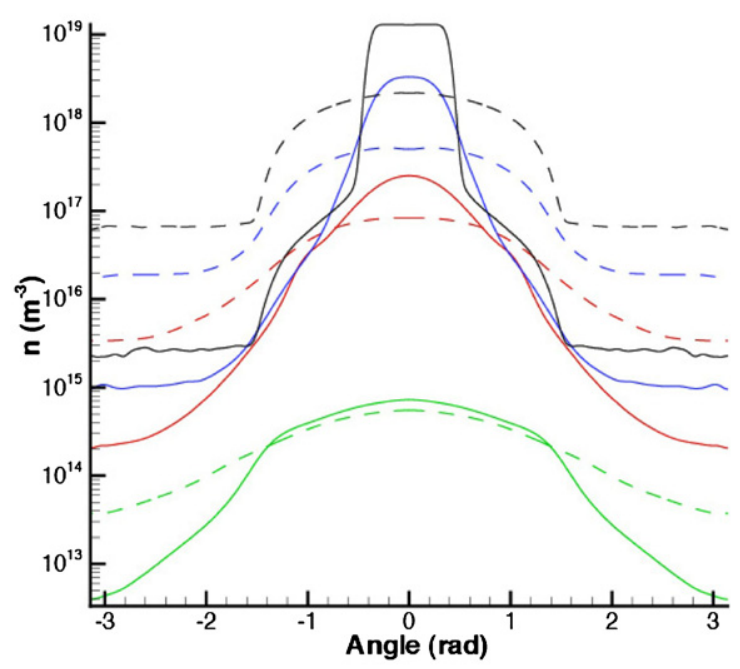

$2.7 \mathrm{AU}$

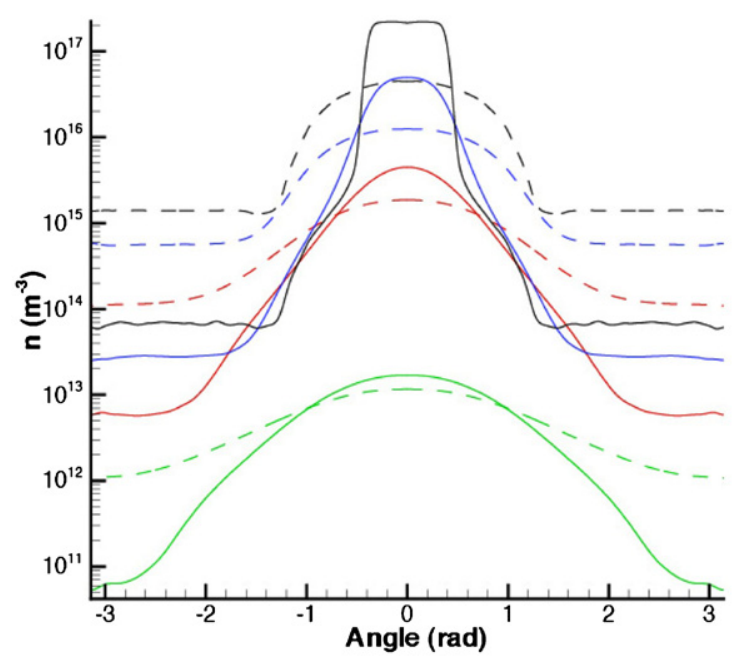

$2.0 \mathrm{AU}$

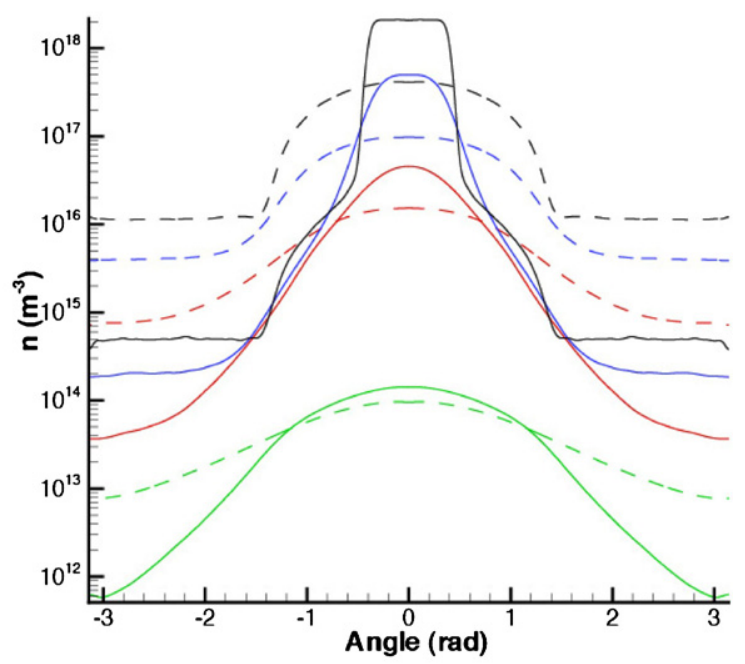

$3.25 \mathrm{AU}$

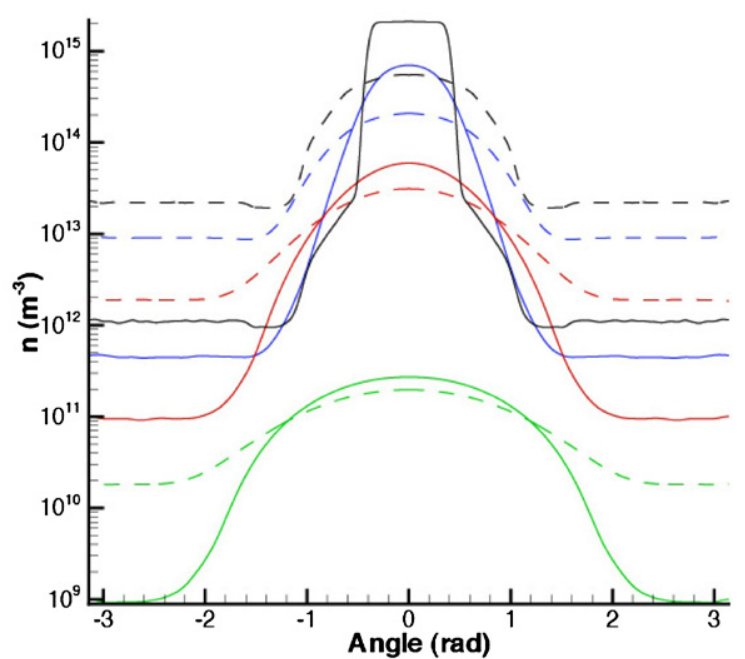

Figure 5. Water vapor density as a function of angle from the center of the active area for the broad and small (4\%) active area models. Shown are cases for four heliocentric distances 1.29, 2.0,2.7, and $3.25 \mathrm{AU}$. The black, blue, red, and green lines are values at $2 \mathrm{~km}, 2.5 \mathrm{~km}, 5 \mathrm{~km}$, and $50 \mathrm{~km}$ from the center of the nucleus, respectively.

feedback from the dust back onto the gas (Crifo \& Rodionov 2000; Tenishev et al. 2011) that would cause significant heating or slowing of the gas flow. These modeled dust results can be rescaled to other dust size distributions, dust/gas mass ratios, bulk densities, and even non-constant bulk densities. With larger production rates, $10^{29}-10^{30}$ molecules $\mathrm{s}^{-1}$, as in very active, long-period comets like 1P/Halley (Combi 1989), the dust acts both as a drag and heating source for the gas (Gombosi et al. 1986).

Figure 7 shows the acceleration region of dust by gas in the coma for the four cases of small active area compared with the broad dayside activity (Tenishev et al. 2011). Because the gas densities from the small active area are much larger than the broad source in the first few $\mathrm{km}$ from the surface, the terminal velocities of particles of all sizes are larger for the small active area. The increase in small dust velocities ranges from a factor of 1.6 for the $1.29 \mathrm{AU}$ perihelion case to $\sim 2$ for the other cases.

The formulation (Gombosi et al. 1986) for calculating the maximum liftable particle is given by the equation of motion of a dust particle entrained in the gas flow as

$$
m_{d} \frac{d v_{d}}{d t}=\sigma_{d} \cdot \frac{1}{2} \cdot C_{d} \cdot n_{d} \cdot m_{d} \cdot\left(v_{g}-v_{d}\right)^{2}-G \frac{m_{d} \cdot M_{c}}{r^{2}}
$$

with the constraint that

$$
\frac{d v_{d}}{d t}=0
$$

This yields the following relationship:

$$
r_{d \max }=\frac{3 \cdot R_{c}^{2} \cdot C_{d} \cdot n_{g} \cdot m_{g} \cdot v_{g}^{2}}{8 \cdot \rho_{d} \cdot G \cdot M_{c}},
$$

where $r=R_{c}=2 \mathrm{~km}$ is the radius of the nucleus, $T_{\text {Knudsen }}=$ $180 \mathrm{~K}$ is the gas temperature in the surface Knudsen layer, $\rho_{c}=300 \mathrm{~kg} \mathrm{~m}^{-3}$ is the comet mass density, $\rho_{d}=1000 \mathrm{~kg} \mathrm{~m}^{-3}$ is the dust grain mass density, $m_{d}=(4 \pi / 3) \rho_{d} r_{d}^{3}$ is the dust 

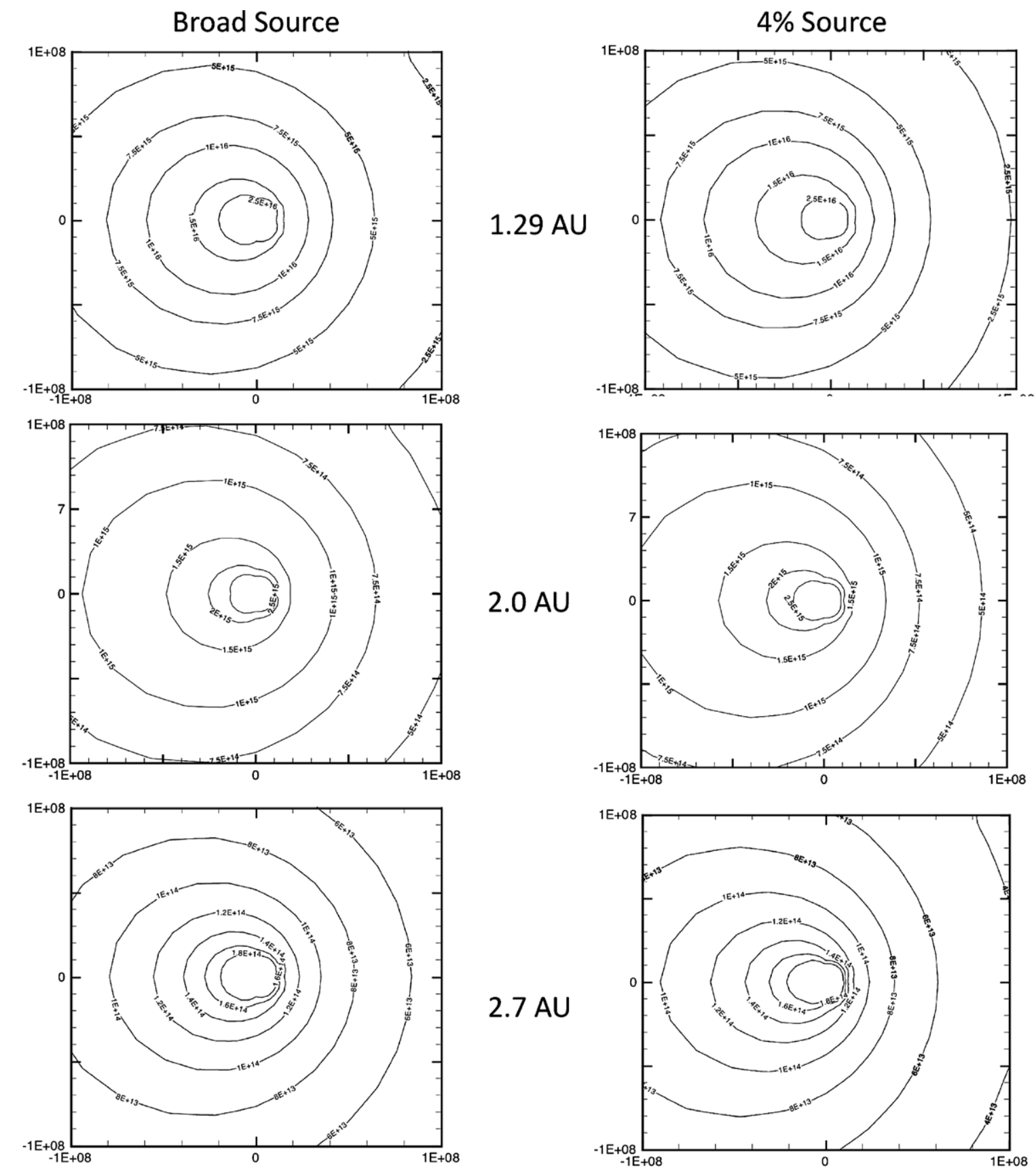

$2.7 \mathrm{AU}$
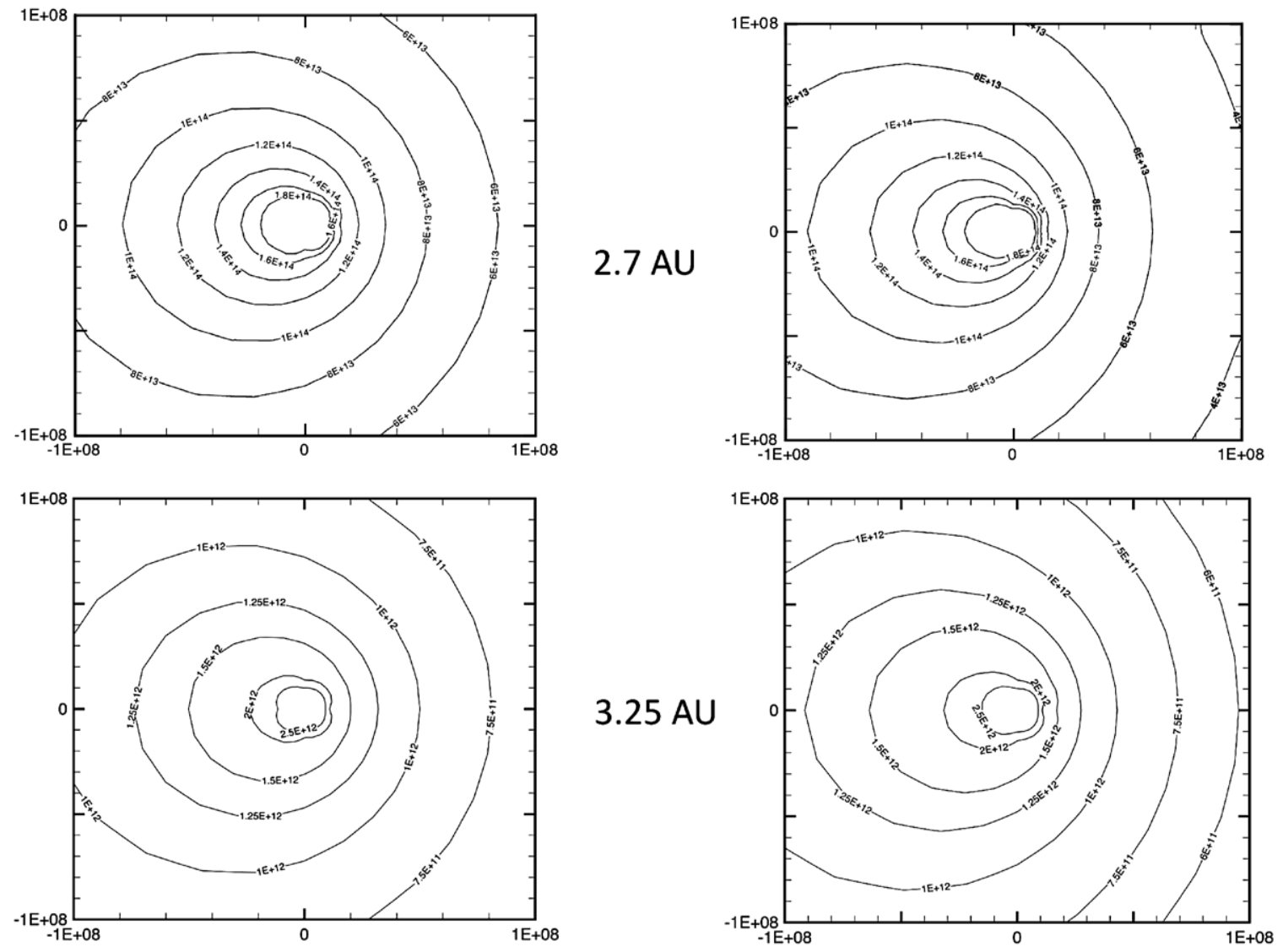

Figure 6. $\mathrm{OH}$ coma produced by broad hemispherical and small active sources. The $\mathrm{OH}$ distribution with the broad hemispherical source distribution model (left) and the $4 \%$ active area model on the right and from top to bottom at heliocentric distances of 1.3, 2.0, 2.7, and 3.25 AU. 

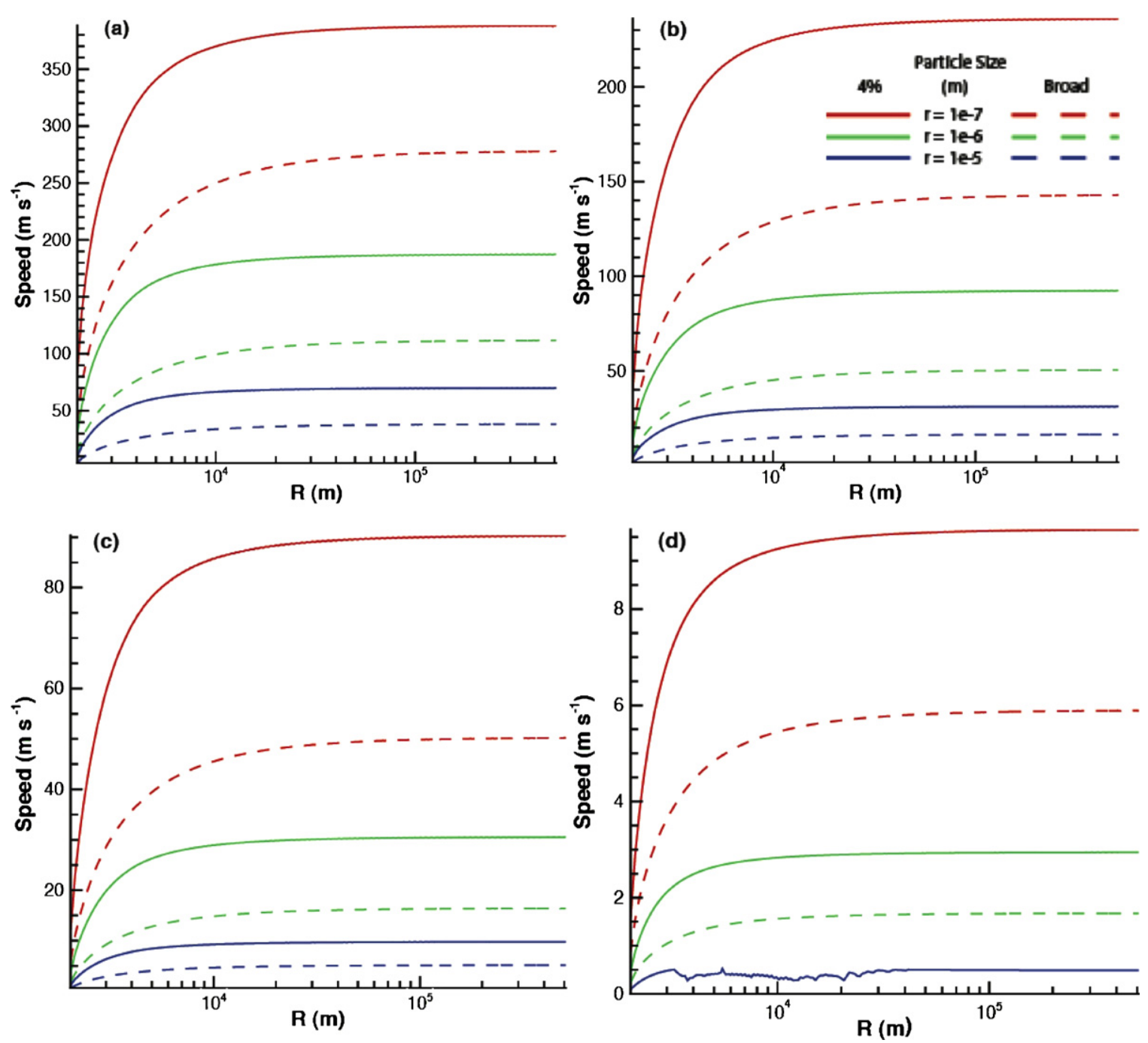

Figure 7. Dust velocities as a function of distance from the center of the nucleus. Shown are dust velocities along the center of the active regions for heliocentric distance cases (a) 1.29, (b) 2.0, (c) 2.7, and (d) $3.25 \mathrm{AU}$ for the broad (dashed) hemispherical source distribution and the small 4\% active area (solid).

Table 2

Modeled Maximum Liftable Dust Sizes

\begin{tabular}{lcc}
\hline \hline Model & \multicolumn{2}{c}{ Particle Radius } \\
\cline { 2 - 3 }$r(\mathrm{AU})$ & Broad Distribution & $4 \%$ Source \\
\hline 1.29 & $7.8 \mathrm{~mm}$ & $42.4 \mathrm{~mm}$ \\
2.0 & $1.7 \mathrm{~mm}$ & $10.3 \mathrm{~mm}$ \\
2.7 & $0.25 \mathrm{~mm}$ & $0.93 \mathrm{~mm}$ \\
3.25 & $5 \mu \mathrm{m}$ & $18 \mu \mathrm{m}$ \\
\hline
\end{tabular}

Note. $r(\mathrm{AU})$ : heliocentric distance in AU.

mass, $\sigma_{d}=2 \pi r_{d}^{2}$ is the dust cross section, $M_{c}=(4 \pi / 3) \rho_{c} R_{c}^{3}=$ $1.01 \cdot 10^{13} \mathrm{~kg}$ is the comet mass, $m_{g}=18 \mathrm{amu}$ is gas molecular mass (assume $\mathrm{H}_{2} \mathrm{O}$ ), $n_{g}$ is the gas number density above the active area, $v_{g}=\sqrt{\left(2 \cdot k \cdot T_{\text {Knudsen }}\right) /\left(\pi \cdot m_{g}\right)}$ is the mean upward expansion velocity, $v_{d}=0$ is initial dust velocity, and $C_{d}=2$ is the drag coefficient.

Table 2 shows the maximum liftable dust size for each of the four cases of small $4 \%$ activity and the previous broad hemispheric activity models. Concentration of the activity into a small area increases the gas density within the first few $\mathrm{km}$ of the nucleus surface, which is precisely where the dust particles reach their terminal velocities (Figure 7). Therefore, it is important to note that in all four cases the small active area lifts particles that are $\sim 3-6$ times larger than the broad hemispherical source, which already has larger maximum liftable dust sizes by factors of 2-3 than the often assumed spherical calculations. The large variation in gas flux around the surface near the nucleus of course will influence the effective dust particle size distribution of dust actually present in the distant coma and tail. It also provides another breaking of the usual simple relationship among particle size, terminal velocity, and the particle $\beta$, the ratio of radiation pressure acceleration to solar gravity, as does particle fragmentation (Combi 1994).

The dust particle density distributions in the inner coma are shown in Figure 8 for the four cases comparing the broad distribution (Tenishev et al. 2011) and small active area models. Color contours for the different models for each heliocentric distance are the same. The first notable difference is that the dust emission right above the peak of the production area is more concentrated for the $4 \%$ active area case than for the broad source even out to $50 \mathrm{~km}$ from the center of the nucleus. This is in contrast to the gas distribution, which becomes nearly as broad by $20 \mathrm{~km}$ from the center of the nucleus in the small active area cases. The reason is that the dust acceleration occurs just above the surface of the nucleus where it is collisionally coupled to the gas and where the gas density is much higher 

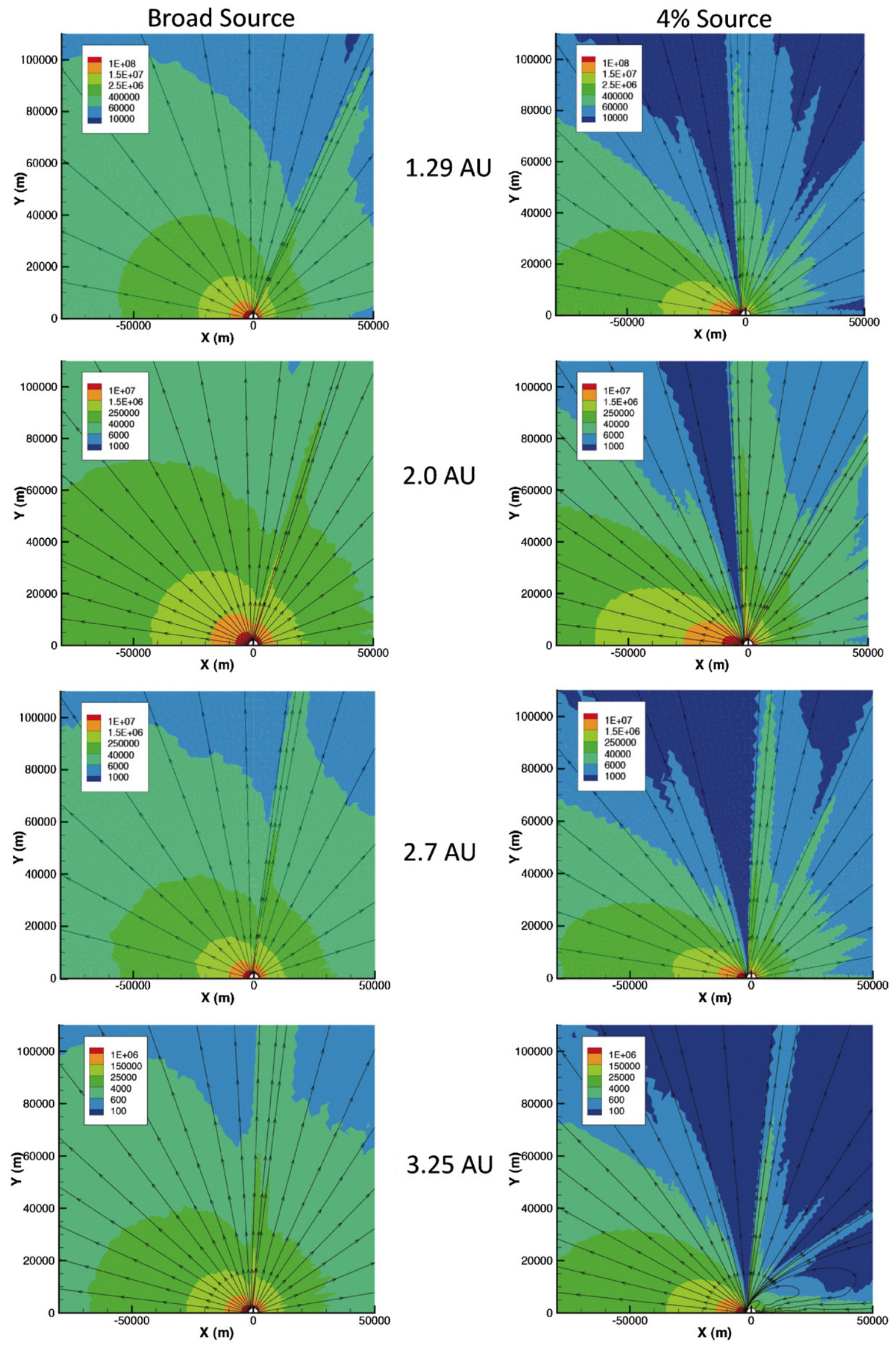

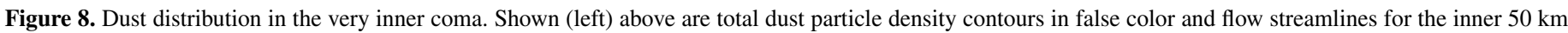

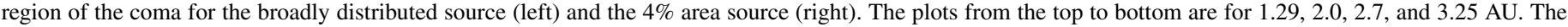
white circles in the lower center of each panel correspond to the $2 \mathrm{~km}$ radius nucleus. 
in the small active area than the broad hemispherical case. The velocity plots in Figure 7 show that the dust particles reach $90 \%$ of terminal velocity $\sim 1-2 \mathrm{~km}$ above the surface, after which they are effectively decoupled from the gas and travel ballistically and continue radially. The gas, on the other hand, continues to expand tangentially, either via gas pressure in the high production rate cases or from free expansion of the initial non-collimated gas velocity distribution function until 5-10 times the nucleus radius when the gas velocities become radial geometrically. Therefore, as seen from a distance, the gas distribution fills nearly the entire hemisphere centered on a small active area but the dust remains highly concentrated on the initial direction from the active area.

The multiple dust spike features from the small active area models shown in Figure 8 form for the same reasons as in the broad hemispherical source models (Tenishev et al. 2011). These are adjacent to steep tangential gradients in the flow, but they appear at different angular locations because of the different emission conditions from the nucleus. As can be seen by the color coding, the densities in the spikes are much lower than those over the main small active area and better illustrated in Figure 9. Their visibility would also depend on favorable orientation with respect to the line of sight.

The concentration of dust over the small active region and its persistence to larger distances is in contrast to the more broad tangential expansion of the underlying gas that drives the dust. This is shown explicitly in Figure 9, which shows the density of gas and dust as a function of angle from the middle of the active region at distances of $2.5,5$, and $50 \mathrm{~km}$ from the center of the nucleus. The black lines show the width of the spot. The full width at half-maximum of the dust emission increases from a half-cone angle of $23^{\circ}$ at 2.5 and $5 \mathrm{~km}$, to only $28^{\circ}$ at $50 \mathrm{~km}$, where the dust is completely decoupled and particles are only moving radially. This is in contrast to the gas, which continues to spread tangentially. Also seen in Figure 9 is the first side dust spike mentioned above, which is more conspicuous in false color plots (Figure 8) than when shown on the absolute scale.

While it has been shown long ago (Finson \& Probstein 1968) that the dust effectively decouples from the gas within a couple of nucleus radii and reaches a size-dependent terminal velocity, previously published model calculations have not been done for a highly restricted active region as has been done here. So, the dust decouples from the gas while it is moving more-orless radially away from the source region. The gas either has substantial tangential pressure (for larger production rates near perihelion) or a substantial tangential velocity distribution (for the small production rates farther from perihelion) and continues to expand tangentially to significant distances from the nucleus and eventually fills most of the hemisphere centered on the active region.

This maintained concentration of dust, essentially straight out from the active area, might provide an alternative, simpler, and more natural explanation to that suggested recently by Belton (2010) for collimated dust outflows in comets, whether $\mathrm{CO}, \mathrm{CO}_{2}$, or water sublimation driven. His Type I jets, appearing as broad fans from a distributed gas emission, normally from the subsolar region of the dayside, are like those produced by the typical 2D axisymmetric (or 3D) calculations as in our broad source models. The picture suggested that his Type II and Type III filamentary jets consist of a source of CO and/or $\mathrm{CO}_{2}$ that is diffusively pumped out into a stream of cold super-volatiles. There is ambient $\mathrm{H}_{2} \mathrm{O}$ sublimation from the surrounding surface that is extremely significant for the process of producing filamentary jets in this picture. The interaction of this flow with the ambient $\mathrm{H}_{2} \mathrm{O}$ atmosphere leads to collimation of the flow. The distinction between Type II and III is that Type II are persistent jets and Type III are episodic. While such a scenario is certainly plausible, the results presented here, having an active source region without a confining ambient gas region, show that the dust remains concentrated over the active region anyway, simply because the dust acceleration occurs fairly close to the nucleus where the gas is concentrated over the active region and is still moving mostly radially outward. The effect is likely enhanced because of the initial rapid expansion of the gas emitted from the surface through the Knudsen layer, where it organizes itself into a slightly supersonic upward moving cooled gas within a few collisional mean free paths. The results shown here are obtained without relying on any specific surface topology such as crevasses, holes, or peculiar dust/gas layers to maintain collimated dust jets as long as the sublimating reservoir contains both gas and dust. Such features may or may not exist in reality and might modify the precise nature of the results obtained. At larger distances (but still fairly close to the nucleus) the dust becomes decoupled from the gas and maintains its initial radial outward motion while the gas continues to expand tangentially to fill much of the hemisphere centered on the location of the active region.

In order to test the generality of the result, we show here a calculation for a much smaller active spot, whose size in angle (or half-cone angle) is typical of the more filamentary dust jets seen in the images of $81 \mathrm{P} /$ Wild 2 by the Stardust camera (Soderblom et al. 2004) or 9P/Tempel 1 by the Deep Impact camera (Farnham et al. 2007). We chose an active spot with the same gas and dust production rate flux per unit area as the perihelion model discussed, but where the spot only has a halfcone angle of $10^{\circ}$. This appears to be the typical size in the spacecraft images. The uniformly distributed background gas source is the same as in the previous models. This means then that the total production rate from the very small active spot is a factor of $\left(1-\cos 10^{\circ}\right) /\left(1-\cos 23^{\circ}\right)$ or $19.1 \%$ of that for the $4 \%$ active area and thus the relative production rate from the active area to the total production rate is $19.7 \%$ (as opposed to the $95 \%$ from the previous models).

Figure 10 shows color contour plots of the distribution of $\mathrm{H}_{2} \mathrm{O}$ gas and dust from a $10^{\circ}$ active spot. Similar to the five times larger $4 \%$ active area, the gas eventually still fills most of the hemisphere centered on the location of the active spot, but the dust remains highly collimated above the spot still with only a small amount of spreading in angle. A plot of total dust density, such as this, is heavily weighted to the smallest particles, which are the ones most strongly coupled to the gas flow. This is illustrated a bit more quantitatively in Figure 11, which shows the gas and dust density at three different distances $(2.5 \mathrm{~km}$, $5 \mathrm{~km}$, and $50 \mathrm{~km}$ ) plotted as a function angle from the location of the active spot. Extending this to a more realistic comet with multiple small active areas would produce a rather diffuse gas distribution with a number of narrow dust jets.

\section{SUMMARY}

The distribution of gas and dust in the coma from a single small active region covering $4 \%$ of the total surface area of the nucleus of a comet, resembling the Rosetta target comet $67 \mathrm{P} /$ Churyumov-Gerasimenko, has been calculated using a kinetic DSMC approach. The gas coma produced expands to fill nearly the entire hemisphere centered on the location of the active area by the time it travels from the $2 \mathrm{~km}$ nucleus radius 


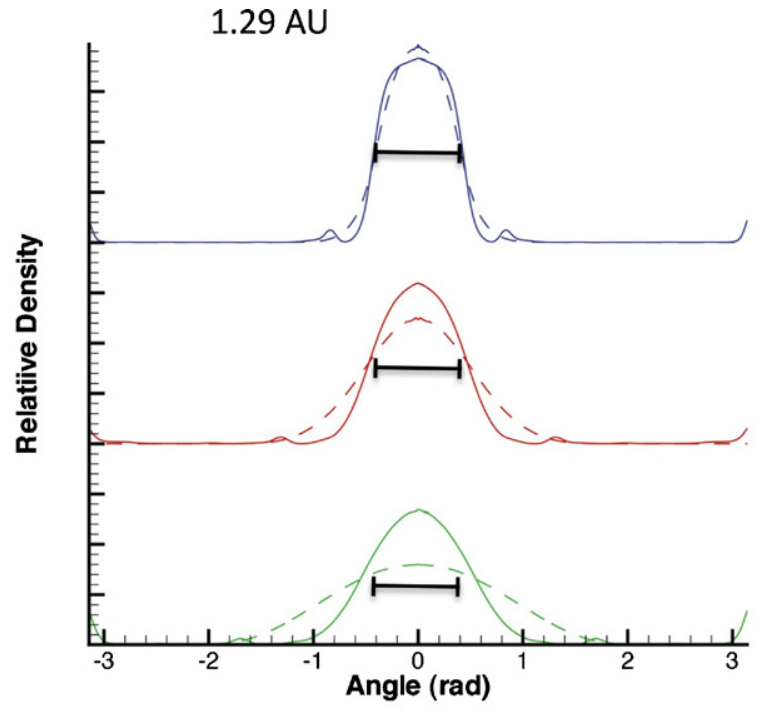

$2.7 \mathrm{AU}$

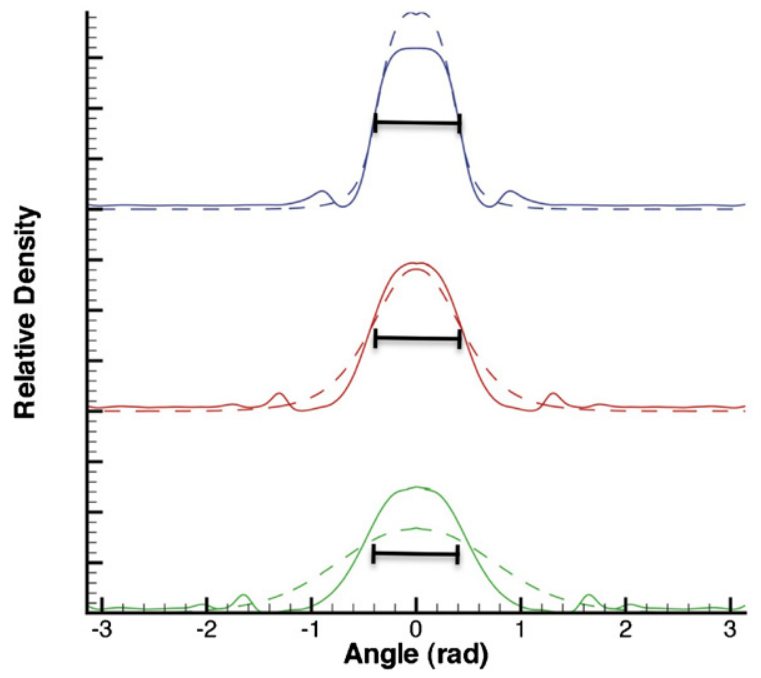

$2.0 \mathrm{AU}$

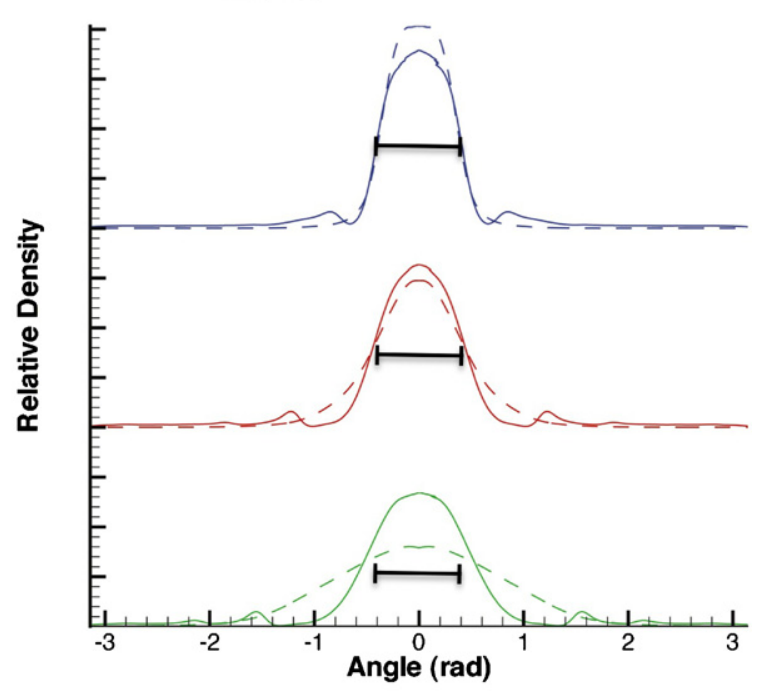

$3.25 \mathrm{AU}$

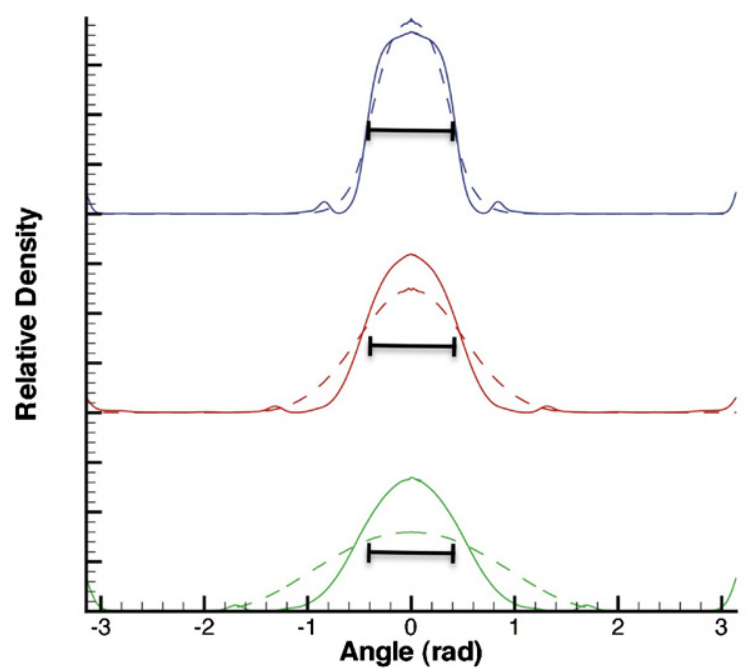

Figure 9. Angular variation of dust and gas flow. Shown are the distributions of gas (dashed) and dust (solid) at $2.5 \mathrm{~km}$ (blue), $5 \mathrm{~km}$ (red), and $50 \mathrm{~km}$ (green) from the center of the nucleus for the small $4 \%$ active area models at heliocentric distances of 1.29, 2.0, 2.7, and 3.25 AU. The black lines show the angular size of the active area on the surface. At $50 \mathrm{~km}$ (green) the dust jet remains highly peaked on the active area with only moderate spreading in the wings.
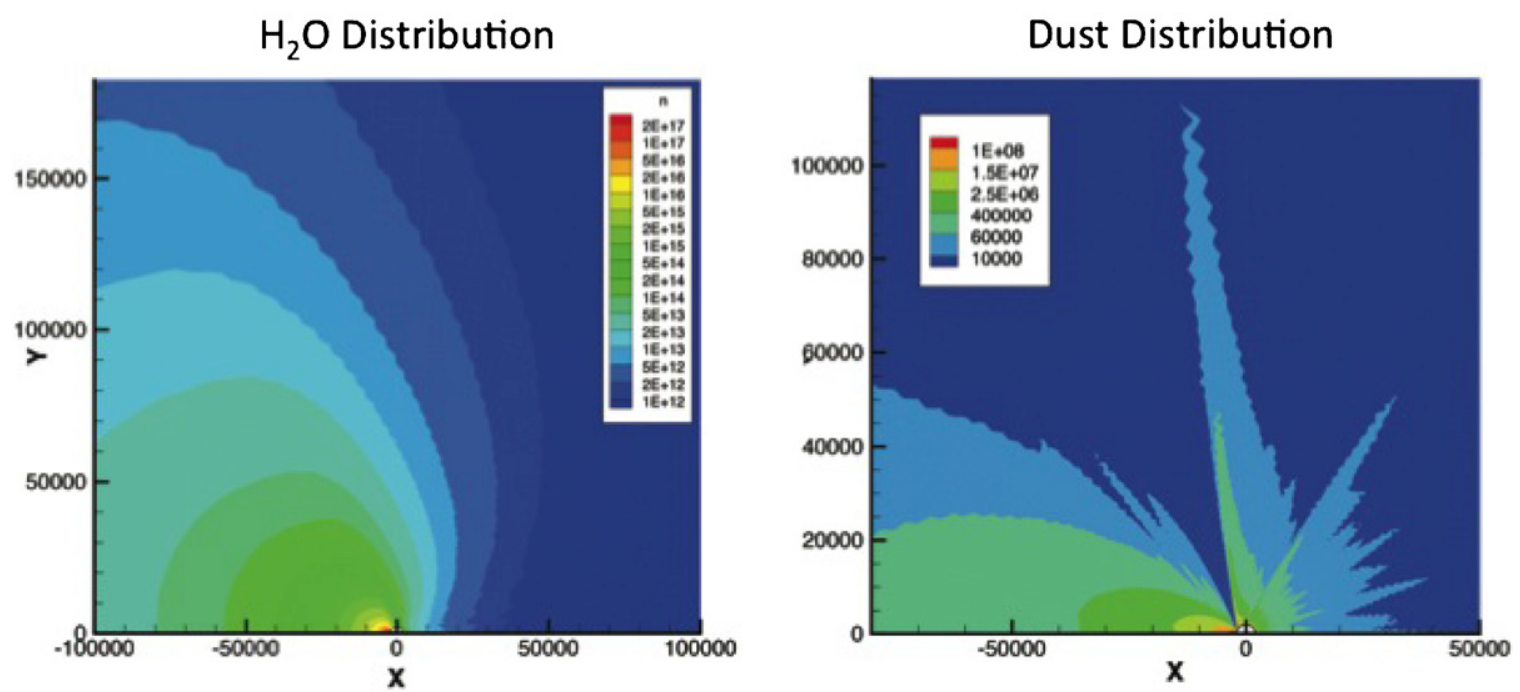

Figure 10. Structure of gas and dust distribution for a filamentary jet. Shown are color contour plots of $\mathrm{H}_{2} \mathrm{O}$ density (left) and dust particle density (right) for the model simulating a filamentary jet from a source region with a half-cone angle of $10^{\circ}$. 


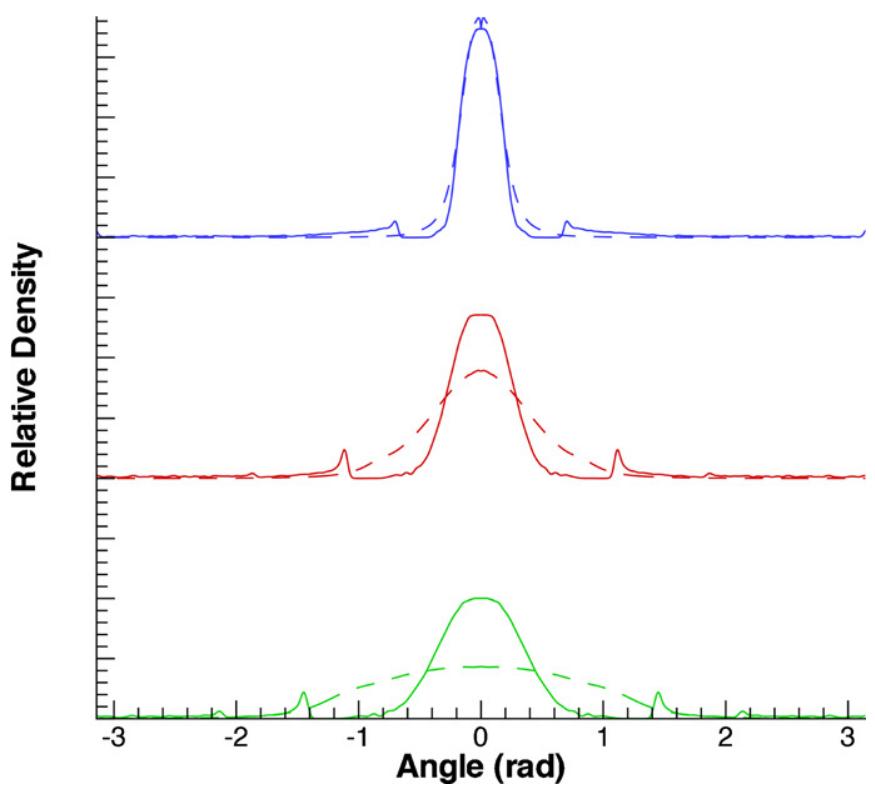

Figure 11. Width of the gas and dust distribution from a $10^{\circ}$ half-cone angle source. The relative water vapor density (solid lines) and dust particle density (dashed lines) are plotted at distance of $2.5 \mathrm{~km}$ (blue), $5 \mathrm{~km}$ (red), and $50 \mathrm{~km}$ (green) for the very small single source region. While the gas still expands to fill much of the hemisphere centered on the location of the original source by $50 \mathrm{~km}$, the dust distribution remains quite narrow.

to a distance of $20 \mathrm{~km}$ from the center of the comet. Thereafter, the gas distribution in the active hemisphere of the coma is otherwise very similar to models where the initial distribution at the surface of the nucleus fills most of a hemisphere. The distribution of daughter radicals produced by such an active area is even more uniform. Realistic complexities (nucleus rotation and time variation of the source) will produce an even less asymmetric observed coma than might be expected.

The dust distribution produced by a small active source is, however, different from a more broadly distributed active area. Dust particles receive most of their acceleration by the gas just above the small active area and are accelerated to much larger terminal velocities. For the small active area, dust terminal velocities over a wide range of sizes were from $60 \%$ to $100 \%$ larger than for a broad mostly hemispherical source. Furthermore, for the largest particles near the gravitational cutoff, the maximum liftable dust sizes were 3-6 times larger from a small active area. Such an effect might be partially responsible for suggestions of the existence of large particles in the dust coma and tail of comet $67 \mathrm{P} /$ Churyumov-Gerasimenko at large heliocentric distances despite rather low overall gas production rates and somewhat dusty values of Af $\rho$ (Fulle et al. 2010; Tozzi et al. 2011).

The decoupling of dust from gas near the nucleus and over a localized active area, combined with the continued tangential expansion of the gas at larger distances might explain the persistence of collimated localized dust features (i.e., jets) over active areas in the presence of more broadly distributed gas in the comae of comets in general. This has been seen in the images of water and dust at 9P/Tempel 1 by the Deep Impact spacecraft (A'Hearn \& Combi 2007), in the concentration of icy grains from the $\mathrm{CO}_{2}$-driven areas from various locations on the nucleus of comet $103 \mathrm{P} /$ Hartley 2 by the EPOXI mission (A'Hearn et al. 2011), and the narrow beams of dust seen in the Deep Space 1 images of comet 19P/Borrelly (Soderblom et al. 2004). Multiple small active regions as seen in some Deep
Impact images of 9P/Temple 1 (Farnham et al. 2007) as well as those seen in the Stardust mission images of $81 \mathrm{P} /$ Wild 2 (Sekanina et al. 2004) should be even more collimated than the model results shown here because the size of those active areas are much smaller than the radius of curvature of the nucleus and there is little divergence to the start of the flow. Conversely, the fans of many radial jets seen emanating from the active lobe of 103P/Hartley 2 in the EPOXI images (A'Hearn et al. 2011) must by analogy result from a cluster of many small active spots driven by $\mathrm{CO}_{2}$.

This work was partially supported by NASA Planetary Atmospheres grant NNX09AB59G and JPL Subcontracts 1266313 and 1266314 under NASA grant NMO710889 to the U.S. Rosetta Project. The computations were made with NAS computer resources at NASA Ames under GID 26135. We acknowledge computer support provided by the Rosetta SGS at ESAC in the framework of Rosetta Working Group X activities.

\section{REFERENCES}

Agarwal, J., Müller, M., \& Grün, E. 2007, Space Sci. Rev., 128, 79

A'Hearn, M. F., Belton, M. J. S., Delamere, W. A., et al. 2011, Science, 332, 1396

A'Hearn, M. F., \& Combi, M. R. 2007, Icarus, 187, 1

A'Hearn, M. F., Millis, R. L., Schleicher, D. G., Osip, D. J., \& Birch, P. V. 1995, Icarus, 118, 223

Belton, M. J. S. 2010, Icarus, 201, 881

Bird, G. A. 1994, Molecular Gas Dynamics and the Direct Simulation of Gas Flows (Oxford: Oxford Univ. Press)

Churyumov, K. I., Gerasimenko, S. I., \& Ya, M. 1969, IAU Circ., 2186

Combi, M. R. 1989, Icarus, 81, 41

Combi, M. R. 1994, AJ, 108, 304

Combi, M. R. 1996, Icarus, 123, 207

Combi, M. R., Bertaux, J.-L., Quemérais, E., \& Mäkinen, J. T. T. 2011, ApJ, 734, L6

Combi, M. R., Harris, W. M., \& Smyth, W. H. 2004, in Comets II, ed. M. C. Festou et al. (Tucson, AZ: Univ. Arizona Press), 523

Combi, M. R., Kabin, K., DeZeeuw, D. L., Gombosi, T. I., \& Powell, K. G. 1997, Earth Moon Planet, 79, 275

Combi, M. R., Mäkinen, J. T. T., Bertaux, J.-L., \& Quemérais, E. 2005, Icarus, 177,228

Crifo, J.-F., Loukianov, G. A., Rodionov, A. V., \& Zakharov, V. V. 2005, Icarus, 176,192

Crifo, J.-F., \& Rodionov, A. V. 1997, Icarus, 129, 72

Crifo, J.-F., \& Rodionov, A. V. 2000, Icarus, 148, 464

Crovisier, J., Colom, P., Gérard, E., Bockelée-Morvan, D., \& Bourgois, G. 2002, A\&A, 393, 1053

Davidsson, B. J. R., Gulkis, S., Alexander, C., et al. 2010, Icarus, 210, 455

Farnham, T., Wellnitz, D. D., Hampton, D. L., et al. 2007, Icarus, 187, 26

Feaga, L. M., A'Hearn, M. F., Sunshine, J. M., Groussin, O., \& Farnham, T. L. 2007, Icarus, 190, 345

Feldman, P., A'Hearn, M., \& Festou, M. 2004, in The New Rosetta Targets, Observations, Simulations and Instrument Performances, ed. L. Colangeli, E. Mazzotta, E. Epifani, \& P. Palumbo (Asrophysics Space Science Library, Vol. 311; Dordrecht: Kluwer), 47

Fink, U. 2009, Icarus, 201, 311

Finson, M. J., \& Probstein, R. F. 1968, ApJ, 154, 327

Fulle, M., Colangeli, L., Agarwal, J., et al. 2010, A\&A, 522, A63

Gombosi, T. I., Cravens, T. E., \& Nagy, A. F. 1986, Rev. Geophys., 24, 667

Groussin, O., Lamy, J. L., \& Besse, S. 2007, BAAS, 39, 485

Hanner, M. S., Tedesco, E., Tokunaga, A. T., et al. 1985, Icarus, 64, 11

Harris, W. M., Combi, M. R., Honeycutt, R. K., \& Mueller, B. E. A. 1997, Science, 277, 676

Keller, H. U. 1990, in Physics and Chemistry of Comets, ed. W. F. Huebner (Berlin: Springer-Verlag), 13

Kidger, M. R. 2003, A\&A, 408, 767

Körösmezey, A., \& Gombosi, T. I. 1990, Icarus, 84, 118

Lamy, P. L., Toth, I., Davidsson, B. J. R., et al. 2007, Space Sci. Rev., 128, 23

Lamy, P. L., Toth, I., Weaver, H., Jorda, L., \& Kaasalainen, M. 2003, BAAS, 35,970

Lisse, C. M., Fernández, Y. R., Kundu, A., et al. 1999, Icarus, 140, 189 
Mäkinen, J. T. T. 2004, in The New Rosetta Targets. Observations, Simulations and Instrument Performances, ed. L. Colangeli, E. Mazzotta Epifani, \& P. Palumbo (Astrophysics Space Science Library, Vol. 311; Dordrecht: Kluwer), 61

Osip, D. J., Schleicher, D. G., \& Millis, R. L. 1992, Icarus, 98, 115

Rickman, H., \& Jorda, L. 1998, Adv. Space Res., 21, 1491

Schleicher, D. G. 2006, Icarus, 181, 442

Schulz, R. 2009, Solar Syst. Res., 43, 343

Schulz, R., Stüwe, J. A., \& Boehnhardt, H. 2004, A\&A, 422, L19

Sekanina, Z., Brownlee, D. E., Economou, T. E., et al. 2004, Science, 304, 1769

Skorov, Yu. V., Markelov, G. N., \& Keller, H. U. 2004, Solar Syst. Res., 38, 455

Soderblom, L. A., Boice, D. C., Britt, D. T., et al. 2004, Icarus, 167, 4
Storrs, A. D., Cochran, A. L., \& Barker, E. S. 1992, Icarus, 98, 163

Tenishev, V., Combi, M. R., \& Davidsson, B. J. R. 2008, ApJ, 685, 659

Tenishev, V., Combi, M. R., \& Rubin, M. 2011, ApJ, 732, 104

Tenishev, V., Combi, M. R., Waite, J. H., \& Teolis, B. 2010, J. Geophys. Res., 115, A09302

Toth, I., Lamy, P., \& Weaver, H. A. 2005, Icarus, 178, 235

Tozzi, G. P., Patriarchi, P., Boehnhardt, H., et al. 2011, A\&A, 531, A54

Valeille, A., Tenishev, V., Bougher, S. W., et al. 2009, J. Geophys. Res., 114 E11005

Waite, J. H., Combi, M. R., Ip, W.-H., et al. 2006, Science, 311, 1419

Weiler, M., Rauer, H., \& Helbert, J. 2004, A\&A, 414, 749

Zakharov, V., Rodionov, A. V., Lukianov, G. A., \& Crifo, J. F. 2009, Icarus, 201, 358 